\title{
Impaired Pulmonary Vascular Adaptation to Exercise in Emphysema without Pulmonary Hypertension - A Proof-of-Concept Study
}

\section{Thibaut Capron ( $\square$ thibaut.capron@gmail.com )}

Assistance Publique - Hôpitaux de Marseille: Assistance Publique Hopitaux de Marseille https://orcid.org/0000-0003-3496-9277

\section{Axel Bartoli}

Assistance Publique - Hôpitaux de Marseille: Assistance Publique Hopitaux de Marseille

Stanislas Rappacchi

Assistance Publique - Hôpitaux de Marseille: Assistance Publique Hopitaux de Marseille

Clarisse Gautier

Assistance Publique - Hôpitaux de Marseille: Assistance Publique Hopitaux de Marseille

\section{Faiza Bettayeb}

Assistance Publique - Hôpitaux de Marseille: Assistance Publique Hopitaux de Marseille

\section{Sophie Morange}

Assistance Publique - Hôpitaux de Marseille: Assistance Publique Hopitaux de Marseille

\section{Maxime Guye}

Assistance Publique - Hôpitaux de Marseille: Assistance Publique Hopitaux de Marseille

Monique Bernard

Aix-Marseille Universite de Provence: Aix-Marseille Universite

\section{Pascal Chanez}

Assistance Publique - Hôpitaux de Marseille: Assistance Publique Hopitaux de Marseille Jean-Yves Gaubert

Assistance Publique - Hôpitaux de Marseille: Assistance Publique Hopitaux de Marseille

\section{Alexis Jacquier}

Assistance Publique - Hôpitaux de Marseille: Assistance Publique Hopitaux de Marseille

\section{Martine Reynaud-Gaubert}

Assistance Publique - Hôpitaux de Marseille: Assistance Publique Hopitaux de Marseille

\section{Research Article}

Keywords: Pulmonary emphysema, hypertension, pulmonary, magnetic resonance imaging, pulmonary disease, chronic obstructive, vascular diseases 
Posted Date: December 29th, 2021

DOI: https://doi.org/10.21203/rs.3.rs-1074821/v1

License: (c) (i) This work is licensed under a Creative Commons Attribution 4.0 International License. Read Full License 


\section{Abstract}

Background: Chronic obstructive pulmonary disease with emphysema lead to respiratory disability beyond bronchial obstruction. The functional impact of pulmonary vascular lesions in emphysema remains unknown. We investigated pulmonary vascular adaptation to exercise in patients with extended emphysema.

Methods: Chest magnetic resonance imaging was used to quantitatively assess right-heart function, pulmonary artery and distal pulmonary blood flow. This was performed at rest and during cycling exercise with a magnetic resonance imaging-compatible cyclo-ergometer. Seven emphysematous patients without pulmonary hypertension were compared to 7 healthy non-smokers matched in gender and age.

Results: At rest, cardio-pulmonary hemodynamics and distal pulmonary vascular parameters were similar in both groups. Intrasubject adaptation to exercise in emphysematous patients was characterized by a higher increase in right-ventricular ejection fraction ( $\triangle \mathrm{RVEF}+8.1 \mathrm{vs}$. $-2.4 \%, P=0.046)$ though a lower rightcardiac output (4.41 vs. $5.79 \mathrm{~L} / \mathrm{min}, P=0.04$ ) at exercise. Accounting for right-cardiac output variation, the distal pulmonary vascular yield index trended to be decreased in patients $\left(\Delta P B F / \Delta Q_{f}-0.78 v s .+18.83 \%\right.$, $P=0.18)$.

Conclusions: Pulmonary vascular adaptation to exercise is impaired in emphysematous patients without identified pulmonary hypertension.

Clinical trial registration NCT 04126616.

\section{Background}

Chronic obstructive pulmonary disease (COPD) is a frequent respiratory condition providing important morbidity and mortality. It was historically described by spirometric indices of bronchial obstruction. Recent large cohort studies among smokers and COPD patients highlighted the heterogeneity of this disease; the bronchial obstruction severity does not always reflect the respiratory disability. For instance, in a population of emphysematous patients without spirometric bronchial obstruction, a significant proportion of cases reported an alteration of the quality of life, exercise hypoxemia or bronchopulmonary exacerbations (1). As a result, the paradigm to guide therapeutic strategies changed in 2017 (2) and is now based on the patients' symptoms (mainly dyspnea and exacerbations).

Although the disease is sometimes accompanied by emphysema, corresponding to alveolo-capillary destruction (3), the pulmonary vascular component has not been extensively studied in COPD (4). Yet early vascular lesions have been shown in COPD patients $(5,6)$. Furthermore, functional alterations of pulmonary circulation were evidenced during exacerbations, both at the microvascular level (7) and in terms of large pulmonary artery vessels (8). They may participate to the respiratory disability beyond bronchial obstruction (9). 
These vascular lesions may result in endothelial dysfunction. Indeed, the vascular endothelium has the skill to adapt the vessel caliber in order to match blood flow to increased needs. Pulmonary endothelial dysfunction can express as suboptimal adaptation of vessels to increase blood flow, which has been initially observed in explanted lungs of patients with severe COPD (10). However this feature, assumed to reflect vascular disease, is difficult to access in vivo.

Magnetic resonance imaging (MRI) is a non-invasive and repeatable technique presenting good contrast for vascular structures. It is widely used in the evaluation of the heart and intrathoracic vessels. Dynamic contrast-enhanced sequences allow to assess quantitatively the pulmonary blood flow in distal capillary regions (11). This method has been used in COPD patients with emphysema, confirming a decreased pulmonary blood flow early in the disease $(12,13)$. However the functional impact of these pulmonary vascular alterations is still poorly known.

In this work, we assumed that pulmonary endothelial dysfunction can be evaluated using dynamic contrast-enhanced MRI. We assessed cardio-pulmonary hemodynamics and distal pulmonary blood flow variations from rest to exercise in emphysematous patients without pulmonary hypertension $(\mathrm{PH})$.

\section{Methods}

This prospective interventional study (NCT 04126616) was conducted following the declaration of Helsinki and our national ethics committee (CPP \#2019-05-045). All subjects gave written informed consent. During the period from October 2019 to June 2020, we prospectively enrolled in this pilot study 7 COPD patients with emphysema and 7 healthy non-smokers matched by gender and age. Inclusion criteria for patients were: age between 40 and 70, diagnosed with COPD, diffuse emphysema on chest computed tomography (CT), forced expiratory volume at $1 \mathrm{~s}$ (FEV1) between 35 and $80 \%$ of predicted values, negative echocardiographic screening for $\mathrm{PH}$. Healthy subjects were recruited following criteria: age between 40 and 70, non-smokers, no background for respiratory disease. Exclusion criteria for both groups were: contra-indication to exercise testing, MRI or Gadolinium-based contrast injection, history of heart disease, background of thoracic surgery with parenchymal resection, intra-thoracic cancer, pregnancy or breast feeding, refusal to be informed in case of incidental finding during the examinations. Healthy subjects were pre-screened with electrocardiogram (ECG), 6 minute walk distance (6MWD) test and blood sample in order to confirm inclusion in the absence of contra-indications. Patients' data were collected from routine medical follow-up. Pulmonary emphysema extent was assessed by an experienced radiologist using Slicer $3 \mathrm{D} \otimes$ and a threshold at $-950 \mathrm{UH}$.

Chest MRI was performed using a commercial imager at $3 \mathrm{~T}$ (Verio ${ }^{\circledR}$, Siemens) and two surface array coils, under continuous monitoring of vital signs. An amagnetic cyclo-ergometer (Lode) was fixed to the table of the MRI system, allowing for cycling exercise. After few minutes of warming-up, the mechanical workload was progressively increased to reach the patient's pre-established target heart rate. This latter was determined by the maximum heart rate measured during 6MWD test, capped at 70\% of the subject's age-predicted maximum heart rate. If oxygen support was indicated from 6MWD test, the titrated oxygen 
flow was delivered to the patient to ensure pulse oximetry $>90 \%$ during MRI exercise. For technical reasons, imaging was performed while stopping cycling; exercise sessions were thus repeated to reach target heart rate before each sequence. At rest and after exercise, routine sequences were used to evaluate left and right heart function, but also the pulmonary artery output from velocity-encoded contrast imaging placed perpendicularly to the pulmonary artery trunk.

We dynamically followed gadolinium-based contrast agent passage through the pulmonary vasculature with repeated images in a coronal plane with a time resolution of one heart cycle (Figure 1). Following the method initially proposed by Oechsner et al. (14), the pulmonary blood flow (PBF) could be mathematically derived from the signal variation in the distal parenchyma and using the simultaneous signal variation measured in the pulmonary artery. Half-dose contrast agent was injected at rest and exercise, allowing for deriving distal PBF variation from rest to exercise, that is distal PBF reserve. Potential differences in right cardiac output variation between the two groups were accounted for by using a distal pulmonary vascular yield index derived as the ratio of PBF variation by right cardiac output variation $\triangle \mathrm{PBF} / \Delta \mathrm{Q}_{\mathrm{f}}$.

Statistical analysis was performed with Excel® (Microsoft). Quantitative variables were expressed as mean \pm standard deviation. Comparisons were assessed with a bilateral paired Student $t$ test and applying Welch's correction when variances were unequal. A $P$ value $<0.05$ was considered as statistically significant.

\section{Results}

The characteristics of the 7 patients and 7 healthy controls included are presented in Table 1. Mean age was $57.4 \pm 6.6$ years in patients' group and 5 were men $(71 \%)$. Controls were matched in age and sex. Blood inflammatory syndrome was significantly higher in patients $(2.56 \pm 1.38 \mathrm{vs} .0 .63 \pm 0.38, P=0.009)$. 
Table 1

Anthropometric, biological and exercise characteristics of the population study ${ }^{\mathrm{a}}$.
\begin{tabular}{|lcll|}
\hline Populations & & \\
\hline Characteristics & Patients & Controls & P \\
\hline Patients & 7 & 7 & \\
\hline Age (years) & $57.4 \pm 6.6$ & $53.3 \pm 7.8$ & 0.31 \\
\hline Male sex (\%) & $5(71.4)$ & $5(71.4)$ & 1 \\
\hline BMI (kg/m $\left.{ }^{2}\right)$ & $23.7 \pm 2.6$ & $25.1 \pm 3.0$ & 0.37 \\
\hline Hematocrit (\%) & $46.1 \pm 2.7$ & $42.7 \pm 3.9$ & 0.08 \\
\hline Blood CRP (mg/L) & $2.56 \pm 1.38$ & $0.63 \pm 0.38$ & 0.009 \\
\hline 6-minute walk test, distance & $499 \pm 92$ & $579 \pm 39$ & 0.09 \\
\hline Maximum heart rate (\% age-predicted) & $61.3 \pm 8.1$ & $64.4 \pm 8.1$ & 0.49 \\
\hline Maximum workload (W) & $54.3 \pm 16.2$ & $69.3 \pm 8.9$ & 0.06 \\
\hline Abbreviations: BMl, body-mass index; CRP, C-reactive protein. & \\
\hline aData are presented as number (percentage) unless otherwise stated. & \\
\hline
\end{tabular}

The 6MWD trended to be lower in patients ( $499 \pm 92 \mathrm{vs.} 579 \pm 39 \mathrm{~m}, P=0.09)$. The exercise intensity during MRI allowed to reach comparable maximum heart rates between the two groups, expressed as a percentage of age-predicted maximum heart rate $(61.3 \pm 8.1 \mathrm{vs}$. $64.4 \pm 8.1 \%, P=0.49)$. The maximum workload trended to be lower in patients ( $54 \pm 16$ vs. $69 \pm 9 \mathrm{~W}, P=0.06$ ).

Disease characteristics of patients are presented in Table 2. They showed moderate bronchial obstruction (mean FEV1 $55 \pm 16 \%$ predicted), important thoracic distension (mean residual volume $174 \pm 47 \%$ predicted) and moderate alteration of alveolar carbon monoxide transfer (mean DLCO $46 \pm 11 \%$ predicted). The mean emphysema extent was $36 \pm 17 \%$ of the total lung parenchyma. Mean room-air resting blood gas oxygen was $71 \pm 8.5 \mathrm{mmHg}$. Two patients $(28.5 \%)$ required oxygen support during exercise. 
Table 2

Disease characteristics in the patient population ${ }^{\mathrm{a}}$.

\begin{tabular}{|ll|}
\hline Patient description & \\
\hline Characteristics & Patients \\
\hline Patients & 7 \\
\hline Dyspnea mMRC & $1.3 \pm 1.1$ \\
\hline Exacerbations ( $\geq 2 /$ year) & $3(42.9 \%)$ \\
\hline Smoking (pack-years) & $33 \pm 23$ \\
\hline FEV1 (\% predicted) & $55 \pm 16$ \\
\hline Residual volume (\% predicted) & $174 \pm 47$ \\
\hline CO transfer (\% predicted) & $46 \pm 11$ \\
\hline Emphysema extent (\%) & $36 \pm 17$ \\
\hline Blood gas oxygen pressure (mmHg) & $71 \pm 8.5$ \\
\hline Oxygen need at exercise & $2(28.5 \%)$ \\
\hline
\end{tabular}

Abbreviations: mMRC, modified medical research council scale; FEV1, forced expiratory volume at $1 \mathrm{~s}$; CO, carbon monoxide.

${ }^{a}$ Data are presented as number (percentage) unless otherwise stated.

Mean parameters for cardio-pulmonary hemodynamics at rest are presented in Table 3. Left and right heart function parameters, and right cardiac output measured in the pulmonary artery, were not significantly different between patients and controls (right cardiac output $3.53 \pm 0.98 \mathrm{~L} / \mathrm{min} v s .3 .53 \pm$ $1.26 \mathrm{~L} / \mathrm{min}, P=0.99)$. 
Table 3

Cardio-pulmonary hemodynamics parameters at rest ${ }^{a}$.

\section{Cardio-pulmonary hemodynamics at rest}

\section{Characteristics}

Patients

Heart rate (bpm)

Left-ventricular function

LVEDV $(\mathrm{mL})$

LVESV $(\mathrm{mL})$

$\operatorname{LVSV}(\mathrm{mL})$

LVEF (\%)

LV mass (g)

Right-ventricular function

$\operatorname{RVSV}(\mathrm{mL})$

RVEF (\%)

Pulmonary artery flow

Antegrade flow (mL/beat)

Right cardiac output (L/min)

Abbreviations: LVEDV, left-ventricular end-diastolic volume; LVESV, left-ventricular end-systolic volume; LVSV, left-ventricular stroke volume; LVEF, left-ventricular ejection fraction; LV mass, left-ventricular mass; RVSV, right-ventricular stroke volume; RVEF, right-ventricular ejection fraction.

${ }^{a}$ Data are presented as numbers unless otherwise stated.

Mean parameters for cardio-pulmonary hemodynamics at exercise are presented in Table 4. The resulting right cardiac output was significantly lower in patients (4.41 \pm 1.13 vs. $5.79 \pm 1.11, P=0.04)$, though heart rate was similar. Intrasubject adaptation to exercise was characterized by a significantly higher increase in right-ventricular ejection fraction (RVEF) in patients, from rest to exercise ( $\triangle \mathrm{RVEF} 8.1 \pm 10.7 v s$. $-2.4 \pm$ $5.4 \%, P=0.046$, Figure 2).
Control

$54 \pm 19$

$64 \pm 17$

0.31

$55 \pm 9$

$61 \pm 4$

0.16

$86 \pm 16$

$93 \pm 23$

0.54
$43 \pm 8$

$52 \pm 13$

$58 \pm 18$

0.49

$3.53 \pm 0.98$

$3.53 \pm 1.26$

0.99

0.2

$48 \pm 5$ 
Table 4

Cardio-pulmonary hemodynamics parameters at exercise ${ }^{\mathrm{a}}$.

\section{Cardio-pulmonary hemodynamics at exercise}

Characteristics

Patients

Heart rate (bpm)

Left-ventricular function

$\operatorname{LVEDV}(\mathrm{mL})$

$\operatorname{LVESV}(\mathrm{mL})$

$\operatorname{LVSV}(\mathrm{mL})$

LVEF (\%)

Right-ventricular function

$\operatorname{RVSV}(\mathrm{mL})$

RVEF (\%)

Pulmonary artery flow

Antegrade flow (mL/beat)

Right cardiac output (L/min)

Abbreviations: LVEDV, left-ventricular end-diastolic volume; LVESV, left-ventricular end-systolic volume; LVSV, left-ventricular stroke volume; LVEF, left-ventricular ejection fraction; LV mass, left-ventricular
mass; RVSV, right-ventricular stroke volume; RVEF, right-ventricular ejection fraction. LVSV, left-ventricular stroke volume; LVEF, left-ventricular ejection fraction; LV mass,
mass; RVSV, right-ventricular stroke volume; RVEF, right-ventricular ejection fraction.

${ }^{a}$ Data are presented as numbers unless otherwise stated.

Control

$P$

7

$79 \pm 14$

$84 \pm 14$

0.54

$98 \pm 26$

$97 \pm 32$

0.98

$36 \pm 14$

$36 \pm 13$

0.97

$62 \pm 17$

$61 \pm 25$

0.95

$63 \pm 7$

$62 \pm 12$

0.79

$50 \pm 15$

$43 \pm 14$

0.34

$51 \pm 7$

$46 \pm 7$

0.15

$57 \pm 18$

$71 \pm 19$

0.2

$4.41 \pm 1.13$

$5.79 \pm 1.11$

0.04

Distal pulmonary blood flow parameters at rest and exercise are shown in Table 5. At rest, mean PBF was comparable between patients and controls $\left(32.0 \pm 18.8 \mathrm{vs}\right.$. $31.6 \pm 29.0 \mathrm{~mL} \cdot \mathrm{min}^{-1} .100 \mathrm{~mL}_{\text {Lung }}{ }^{-1}$ for left lung, $P=0.97)$. Intrasubject PBF variation from rest to exercise trended to be lower in patients ( $\triangle \mathrm{PBF} 15.1$ \pm 10.3 vs. $44.6 \pm 55.1 \mathrm{~mL} \cdot \mathrm{min}^{-1} .100 \mathrm{~mL}_{\text {Lung }}{ }^{-1}, P=0.21$ ). Accounting for the right cardiac output variation from rest to exercise which was found lower in patients, a tendency to a lower distal pulmonary vascular yield index was evidenced $\left(\triangle \mathrm{PBF} / \Delta \mathrm{Q}_{\mathrm{f}}-0.78 \pm 30.64 v s\right.$. $18.83 \pm 19.36 \%, P=0.18$, Figure 3$)$. 
Table 5

Distal pulmonary blood flow parameters at rest and exercise ${ }^{\mathrm{a}}$.

\section{Distal pulmonary blood flow}

\begin{tabular}{|c|c|c|c|}
\hline Characteristics & Patients & Controls & $P$ \\
\hline Patients & 7 & 7 & \\
\hline \multicolumn{4}{|l|}{ Rest parameters } \\
\hline PBF left lung (mL.min $\left.{ }^{-1} \cdot 100 \mathrm{~mL}_{\text {Lung }}{ }^{-1}\right)$ & $32.0 \pm 18.8$ & $31.6 \pm 29.0$ & 0.97 \\
\hline PBF right lung $\left(\mathrm{mL} \cdot \mathrm{min}^{-1} .100 \mathrm{~mL}_{\text {Lung }}{ }^{-1}\right)$ & $32.3 \pm 26.9$ & $30.3 \pm 18.7$ & 0.87 \\
\hline \multicolumn{4}{|l|}{ Exercise parameters } \\
\hline PBF left lung (mL.min $\left.{ }^{-1} .100 m L_{\text {Lung }^{-1}}{ }^{-1}\right)$ & $43.1 \pm 24.5$ & $63.1 \pm 46.2$ & 0.34 \\
\hline PBF right lung (mL.min $\left.{ }^{-1} .100 \mathrm{~mL}_{\text {Lung }}{ }^{-1}\right)$ & $42.2 \pm 20.6$ & $72.8 \pm 54.2$ & 0.2 \\
\hline \multicolumn{4}{|l|}{ Intrasubject variation rest-exercise } \\
\hline$\Delta \mathrm{PBF}\left(\mathrm{mL} \cdot \mathrm{min}^{-1} .100 \mathrm{~mL}_{\text {Lung }^{-1}}\right)$ & $15.1 \pm 10.3$ & $44.6 \pm 55.1$ & 0.21 \\
\hline Index $\Delta \mathrm{PBF} / \Delta \mathrm{Q}_{\mathrm{f}}(\%)$ & $-0.78 \pm 30.64$ & $18.83 \pm 19.36$ & 0.18 \\
\hline \multicolumn{4}{|c|}{ Abbreviations: PBF, pulmonary blood flow; $\Delta Q_{f}$, right cardiac output variation from rest to exercise. } \\
\hline
\end{tabular}

\section{Discussion}

This proof-of-concept study highlights the functional pulmonary vascular deficit of exercise adaptation in emphysematous patients without clinical and echocardiographic signs of $\mathrm{PH}$. Though resting hemodynamics were similar with healthy non-smokers, our results emphasize a significantly different adaptation of the right heart at exercise, characterized by a larger increase in RVEF and a lower right cardiac output.

In this work, patients were recruited based on the presence of extended emphysema on chest CT (mean $36 \%$ of parenchymal volume) and showed moderate bronchial obstruction (mean FEV1 55\% predicted), severe thoracic distension (mean residual volume 174\% predicted) and moderate alteration of alveolar gas transfer (mean DLCO 46\% predicted), compatible with a "pulmonary vascular phenotype" for which 
the respiratory disability exceeds the bronchial obstruction level (15). Blood inflammatory syndrome was significantly higher in patients, according to the known chronic inflammation in COPD (16).

The exercise performance was lower in patients (6MWD test or maximum workload), in relation with the expected respiratory disability. However the hemodynamics exercise intensity was similar in both groups ; the maximum heart rate at exercise (expressed as a percentage of age-predicted maximum heart rate) was not different between patients and controls.

Hemodynamic adaptation to exercise was characterized by a larger increase in RVEF in patients. Such variation was not observed for the left-sided heart. However the resulting right cardiac output measured in the pulmonary artery trunk was lower in patients at exercise. This adaptation profile suggests an impaired pulmonary circulation downstream compatible with elevated pulmonary vascular resistances. This anomaly is revealed only at exercise and reminds an exercise-induced pulmonary artery hypertension profile (17). Moreover, it may not be related with hypoxia-induced lesions as mean room-air resting blood gas oxygen was $71 \mathrm{mmHg}$ in patients.

Regarding distal PBF, rest measurements found good right-left homogeneity at the group level. Importantly, resting distal PBF was similar between the two groups although rarefaction of the pulmonary parenchyma could have created a difference in emphysematous patients. The PBF measurement method was intrinsically sensitive to the parenchymal vascular density (flow given for $100 \mathrm{~mL}$ of lung) and avoided such pitfall.

The intrasubject distal vascular yield index reflected the ability to perfuse pulmonary distal regions by increasing right cardiac output (Figure 3). Though statistical significance was not reached, mean index showed striking difference, being $<1 \%$ in patients and $19 \%$ in controls. To the best of our knowledge, this is the first study reporting functional pulmonary vascular impairment at exercise in vivo in emphysematous patients. This may explain one of the mechanisms causing the respiratory disability in patients with a "pulmonary vascular phenotype".

Besides the proof-of-concept nature of this study, main limitations of this protocol are technical. Assessment of PBF requires manual delineation of regions of interest on series of time frames and subsequent adjustable fitting procedure. This is time consuming and could be improved with automatized and standardized detection processes in the future. Inclusion of patients with diagnosed pulmonary hypertension may bring better understanding of right-heart hemodynamics at exercise, however MRI supine exercise may be challenging in such population.

\section{Conclusions}

In conclusion, this study reports an impaired pulmonary vascular adaptation to exercise in emphysematous patients without $\mathrm{PH}$, as measured with chest MRI. These vascular alterations may participate to respiratory disability. Furthermore, the associated right-heart adaptation profile suggests 
isolated exercise pulmonary vascular impairment which could be a therapeutic target to prevent emphysema evolution towards pulmonary hypertension.

\section{Abbreviations}

Chronic obstructive pulmonary disease (COPD)

Magnetic resonance imaging (MRI)

Pulmonary hypertension $(\mathrm{PH})$

Computed tomography (CT)

Forced expiratory volume at $1 \mathrm{~s}$ (FEV1)

Electrocardiogram (ECG)

6 minute walk distance (6MWD)

Pulmonary blood flow (PBF)

Diffusing capacity of the lung for carbon monoxide (DLCO)

Right-ventricular ejection fraction (RVEF)

Arbitrary units (AU)

Body-mass index (BMI)

C-reactive protein (CRP)

modified medical research council scale (mMRC)

Left-ventricular end-diastolic volume (LVEDV)

Left-ventricular end-systolic volume (LVESV)

Left-ventricular stroke volume (LVSV)

Left-ventricular ejection fraction (LVEF)

Left-ventricular (LV)

Right-ventricular stroke volume (RVSV)

\section{Declarations}


This study was conducted following ethics standards from the declaration of Helsinki and french national ethics committee. All subjects gave written informed consent.

This study was supported by a grant from Assistance Publique Hôpitaux de Marseille (AORC junior 2018).

The authors declare that they have no competing interests.

TC, MG, MB, JYG, AJ and MRG contributed to the study concept and design. TC, SR, CG, FB, SM, PC contributed to the acquisition of data. TC, $A B$ contributed to data analysis. TC, $A B, S R$ and MRG contributed to data interpretation. TC and MRG contributed to drafting manuscript. MB, AJ and MRG contributed to critically revising manuscript for important intellectual content. All authors read and approved the final manuscript.

\section{References}

1. Alcaide AB, Sanchez-Salcedo P, Bastarrika G, Campo A, Berto J, Ocon MDM, et al. Clinical Features of Smokers With Radiological Emphysema But Without Airway Limitation. Chest. févr 2017;151(2):358-65.

2. Vogelmeier CF, Criner GJ, Martinez FJ, Anzueto A, Barnes PJ, Bourbeau J, et al. Global Strategy for the Diagnosis, Management, and Prevention of Chronic Obstructive Lung Disease 2017 Report. GOLD Executive Summary. Am J Respir Crit Care Med. 2017; 195(5):557-82.

3. Liebow AA. Pulmonary emphysema with special reference to vascular changes. Am Rev Respir Dis. juill 1959;80(1, Part 2):67-93.

4. Blanco I, Piccari L, Barberà JA. Pulmonary vasculature in COPD: The silent component. Respirol Carlton Vic. 2016;21(6):984-94.

5. Peinado VI, Pizarro S, Barberà JA. Pulmonary vascular involvement in COPD. Chest. oct 2008; 134(4):808-14.

6. Clarenbach CF, Senn O, Sievi NA, Camen G, van Gestel AJR, Rossi VA, et al. Determinants of endothelial function in patients with COPD. Eur Respir J. nov 2013;42(5): 1194-204.

7. Barberà JA, Roca J, Ferrer A, Félez MA, Díaz O, Roger N, et al. Mechanisms of worsening gas exchange during acute exacerbations of chronic obstructive pulmonary disease. Eur Respir J. juin 1997; 10(6):1285-91.

8. Wells JM, Washko GR, Han MK, Abbas N, Nath H, Mamary AJ, et al. Pulmonary arterial enlargement and acute exacerbations of COPD. N Engl J Med. 6 sept 2012; 367(10): 913-21.

9. Capron T, Bourdin A, Perez T, Chanez P. COPD beyond proximal bronchial obstruction: phenotyping and related tools at the bedside. Eur Respir Rev Off J Eur Respir Soc. 30 juin 2019; 28(152).

10. Dinh-Xuan AT, Higenbottam TW, Clelland CA, Pepke-Zaba J, Cremona G, Butt AY, et al. Impairment of endothelium-dependent pulmonary-artery relaxation in chronic obstructive lung disease. $\mathrm{N}$ Engl $\mathrm{J}$ Med. 30 mai 1991; 324(22):1539-47. 
11. Hatabu H, Tadamura E, Levin DL, Chen Q, Li W, Kim D, et al. Quantitative assessment of pulmonary perfusion with dynamic contrast-enhanced MRI. Magn Reson Med. déc 1999; 42(6):1033-8.

12. Hueper K, Parikh M, Prince MR, Schoenfeld C, Liu C, Bluemke DA, et al. Quantitative and Semiquantitative Measures of Regional Pulmonary Parenchymal Perfusion by Magnetic Resonance Imaging and their Relationships to Global Lung Perfusion and Lung Diffusing Capacity - The MESA COPD Study. Invest Radiol. avr 2013; 48(4):223-30.

13. Hueper K, Vogel-Claussen J, Parikh MA, Austin JHM, Bluemke DA, Carr J, et al. Pulmonary Microvascular Blood Flow in Mild Chronic Obstructive Pulmonary Disease and Emphysema. The MESA COPD Study. Am J Respir Crit Care Med. 1 sept 2015; 192(5) :570-80.

14. Oechsner M, Mühlhäusler M, Ritter $C O$, Weininger $M$, Beissert $M$, Jakob PM, et al. Quantitative contrast-enhanced perfusion measurements of the human lung using the prebolus approach. $\mathrm{J}$ Magn Reson Imaging. 2009;30(1):104-11.

15. Kovacs G, Agusti A, Barberà JA, Celli B, Criner G, Humbert M, et al. Pulmonary Vascular Involvement in Chronic Obstructive Pulmonary Disease. Is There a Pulmonary Vascular Phenotype? Am J Respir Crit Care Med. 15 oct 2018;198(8):1000-11.

16. Barnes PJ. Mediators of chronic obstructive pulmonary disease. Pharmacol Rev. déc 2004; 56(4):515-48.

17. Tolle JJ, Waxman AB, Van Horn TL, Pappagianopoulos PP, Systrom DM. Exercise-induced pulmonary arterial hypertension. Circulation. 18 nov 2008; 118(21):2183-9.

\section{Figures}



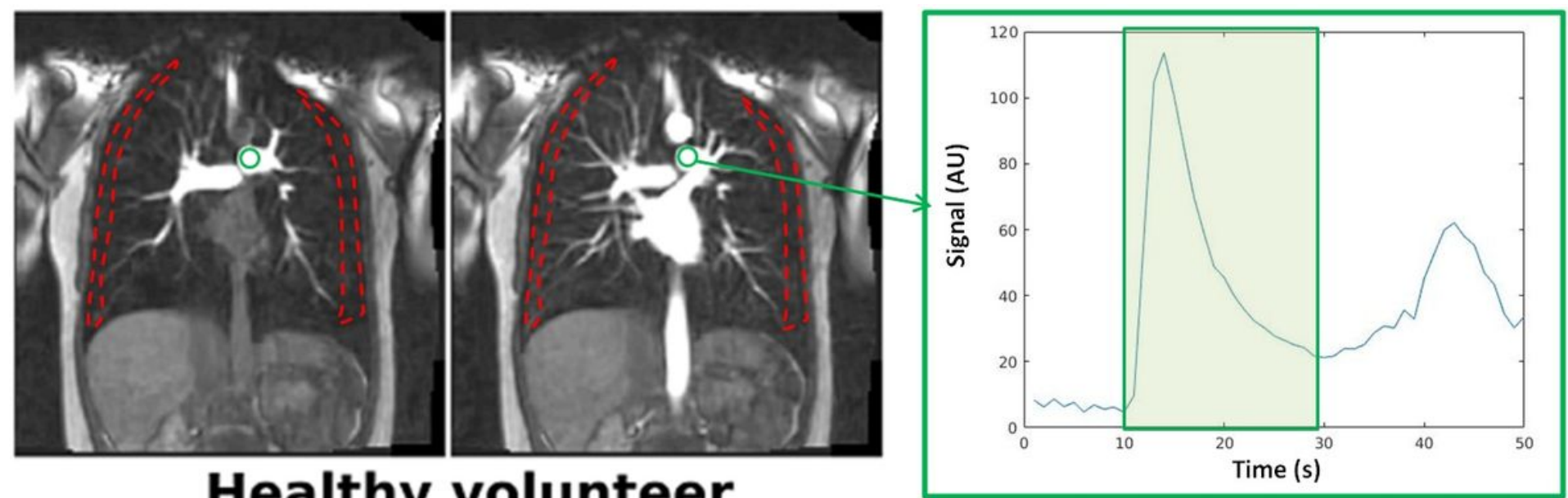

Healthy volunteer
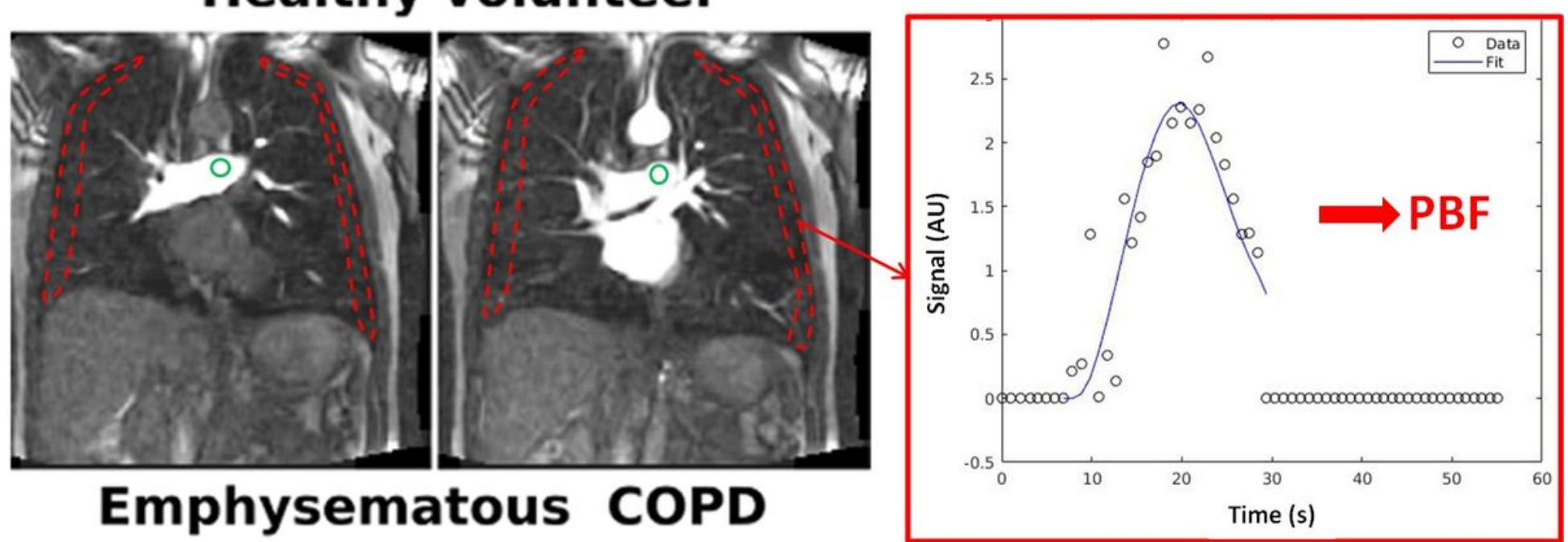

Emphysematous COPD

Figure 1

Chest magnetic resonance images obtained at pulmonary artery (left) and pulmonary veins (right) times. Regions of interest placed in the pulmonary artery (green) and in the distal lung (dashed red) allow reconstruction of the signal time course during contrast agent passage. First-pass signal measured in the pulmonary artery region (green curve frame) is used to mathematically derive fitting curve (red curve frame) of the distal lung signal (open dots) and assess the pulmonary blood flow.

Abbreviations: AU, arbitrary units; COPD, chronic obstructive pulmonary disease; PBF, pulmonary blood flow. 


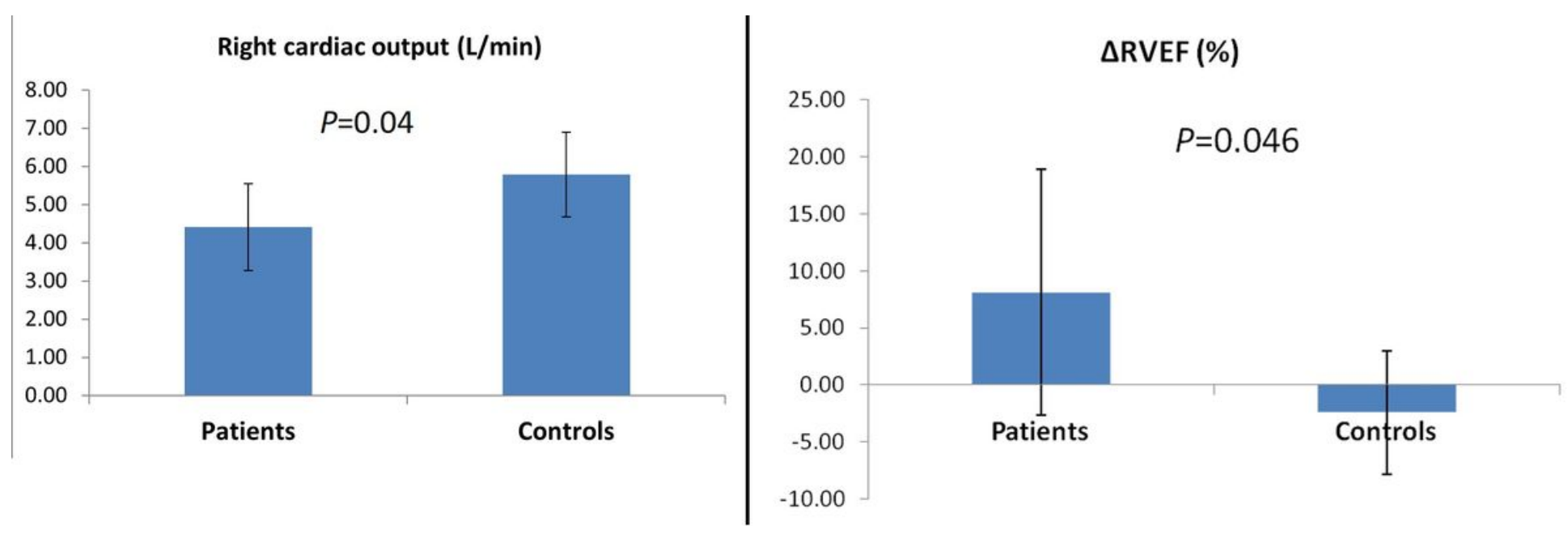

Figure 2

A: Exercise right cardiac output in patients (left bar) and controls (right bar). B: Intrasubject rightventricular ejection fraction variation from rest to exercise, in patients (left bar) and controls (right bar). Abbreviations: $\triangle \mathrm{RVEF}$, right-ventricular ejection fraction variation from rest to exercise.

\section{Index $\Delta P B F / \Delta Q_{f}(\%)$}

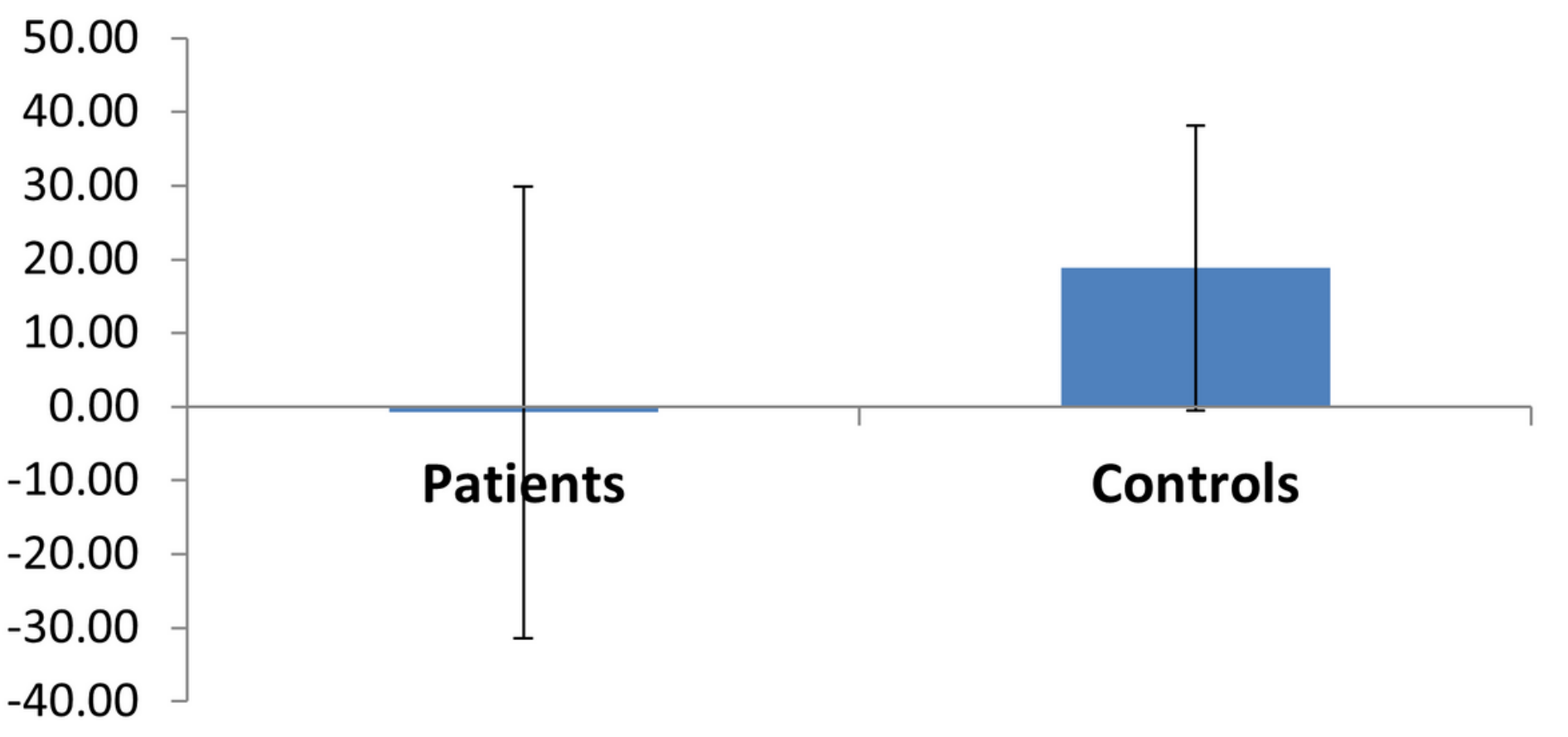

\section{Figure 3}

Intrasubject distal vascular yield index in patients (left bar) and controls (right box). 
Abbreviations: $\triangle \mathrm{PBF}$, pulmonary blood flow variation from rest to exercise; $\Delta \mathrm{Q}_{\mathrm{f}}$, right cardiac output variation from rest to exercise. 